\title{
Mild Traumatic Brain Injury in Translation
}

\author{
Harvey S. Levin ${ }^{1,2}$ and Claudia S. Robertson ${ }^{3}$
}

\begin{abstract}
This Introduction to a Special Issue on Mild Traumatic Brain Injury (mTBI) highlights the methodological challenges in outcome studies and clinical trials involving patients who sustain mTBI. Recent advances in brain imaging and portable, computerized cognitive tasks have contributed to protocols that are sensitive to the effects of mTBI and efficient in time for completion. Investigation of civilian $\mathrm{mTBI}$ has been extended to single and repeated injuries in athletes and blastrelated mTBI in service members and veterans. Despite differences in mechanism of injury, there is evidence for similar effects of acceleration-deceleration and blast mechanisms of mTBI on cognition. Investigation of repetitive mTBI suggests that the effects may be cumulative and that repeated mTBI and repeated subconcussive head trauma may lead to neurodegenerative conditions.

Although animal models of mTBI using cortical impact and fluid percussion injury in rodents have been able to reproduce some of the cognitive deficits frequently exhibited by patients after mTBI, modeling post-concussion symptoms is difficult. Recent use of closed head and blast injury animal models may more closely approximate clinical mTBI. Translation of interventions that are developed in animal models to patients with mTBI is a priority for the research agenda. This Special Issue on mTBI integrates basic neuroscience studies using animal models with studies of human mTBI, including the cognitive sequelae, persisting symptoms, brain imaging, and host factors that facilitate recovery.
\end{abstract}

Key words: adult brain injury, military injury, traumatic brain injury

\section{Introduction}

W ITH RECENT PROGRESS IN LABORATORY RESEARCH and clinical investigations concerning the neural mechanisms of mild traumatic brain injury (mTBI), it is timely to review research on these frequently occurring injuries in civilian and military populations. The prospective, longitudinal investigation of recovery from mTBI arising from closed head trauma in civilians, ${ }^{1}$ studies of blast-related mTBI in the military, ${ }^{2,3}$ and proliferation of sports concussion research ${ }^{4,5}$ provide a rationale for publication of this Special Issue. The impact of mTBI on public health is significant, because it accounts for approximately $85 \%$ of the 1.7 million civilian cases of TBI that are reported annually in the United States. ${ }^{6}$ Combat-related mTBI, including blast and non-blast mechanisms, has emerged as the "signature injury" during the recent wars in Iraq and Afghanistan, reflecting its occurrence in $15 \%$ to $23 \%$ of service members. ${ }^{7,8}$

Laboratory scientists have developed animal models of blastrelated $\mathrm{mTBI}^{3}{ }^{3}$ and investigators have reported the results of post-deployment neurocognitive testing and brain imaging. ${ }^{9-12}$ Recently, prospective investigation of subacute mTBI in service members has produced novel findings about the effects of these injuries on $\operatorname{cognition}^{13}$ and on the integrity of cerebral white matter tracts using diffusion tensor imaging (DTI). ${ }^{14}$

\section{Civilian mTBI}

With the exception of approximately $2 \%$ of patients whose neurologic status deteriorates because of delayed complications of an apparent mTBI and patients with severe, concomitant injury to other body regions, survival is nearly assured after mTBI. ${ }^{6}$ Prospective, longitudinal studies that have recruited civilian mTBI patients within a few days after injury have reported that cognitive function generally recovers within 3 months to a level comparable to control groups without mTBI. ${ }^{15}$ A subgroup of mTBI patients, ${ }^{16}$ however, estimated to be as high as $15 \%$, evidence persistent post-concussion symptoms (PCS) including somatic (e.g., headaches), cognitive (e.g., memory and attention problems), and emotional (e.g., depression) symptoms and other features that have been diagnosed as "postconcussion disorder" or "post-concussion syndrome." The size of this subgroup has been disputed, especially when it is based on data from specialty clinics treating chronic patients with persistent symptoms who may not be representative of the mTBI population. Advances in brain imaging and improved methodology in studying cognitive and behavioral recovery from mTBI have implicated the contribution of pre-existing and co-morbid conditions to symptoms persisting longer than 3 months after mTBI. ${ }^{1}$

In comparison with the general population, patients sustaining traumatic injury have a higher rate of risk factors, including

\footnotetext{
${ }^{1}$ Department of Veterans Affairs, Neurorehabilitation: Neurons to Networks Traumatic Brain Injury Center of Excellence (project \#B6812C), Michael E. DeBakey Veterans Affairs Medical Center, Houston, Texas.

Departments of ${ }^{2}$ Physical Medicine and Rehabilitation and ${ }^{3}$ Neurosurgery, Baylor College of Medicine, Houston, Texas.
} 
substance abuse, impulsivity, and other behavioral disturbances that potentially affect functional and neurobehavioral outcomes. ${ }^{17}$ More than half of adults seen in level 1 trauma centers for acute mTBI have detectable levels of alcohol or drugs suggesting intoxication, ${ }^{18}$ but further screening is necessary to identify a subgroup whose substance dependence interferes with daily functioning, a serious confound in outcome studies. ${ }^{15}$

To differentiate the effects of mTBI from host factors predisposing to traumatic injury and control for nonspecific effects of a traumatic injury, ${ }^{15}$ some investigators have included a comparison group of patients who sustained an orthopedic injury (OI) whose demographic features are comparable to those of mTBI patients. ${ }^{19-21}$ Few studies have differentiated mTBI patients whose computed tomography (CT) findings show cortical contusions or other lesions, a complication that can adversely impact cognitive recovery. ${ }^{22}$

\section{Sports concussion and effects of repetitive injury}

Research on detecting the subacute effects of sports concussion and characterizing its recovery has proliferated. Aided by development of portable devices for cognitive assessment, helmetmounted accelerometers to measure traumatic forces, ${ }^{23}$ and access to athletic teams for pre-season and post-concussion assessments, investigators have characterized the trajectory for recovery from a single, uncomplicated concussion and initiated studies of repetitive head impacts and repeated concussions. ${ }^{4,5}$

Since Gronwall and Wrightson ${ }^{24}$ reported in 1975 an initial study of repeated mTBI suggesting the possibility of cumulative effects, widespread interest in repetitive mTBI has been generated by the proliferation of studies on sports concussion, increasing popularity of boxing and mixed martial arts, and the sequelae of mTBI sustained by military personnel during the wars in Iraq and Afghanistan. ${ }^{25}$ Using telemetry to measure head impacts sustained by collegiate athletes over the course of a season, McAllister and associates ${ }^{26}$ found evidence for cumulative effects of head impacts on cognition. Twenty-four percent of the players had postseason verbal learning scores that were lower than expected based on their preseason performance, whereas this occurred in only $3.6 \%$ of athletes engaged in non-contact sports. Within the group who played a contact sport, greater exposure to head impacts was associated with slower reaction time on ImPACT testing. Further investigation of the long-term effects of repetitive head impacts is clearly indicated, especially with inclusion of brain imaging.

\section{Chronic traumatic encephalopathy (CTE)}

CTE is a neurodegenerative brain disease that is thought to be caused at least in part by repetitive brain trauma. ${ }^{27}$ The reader is referred to recent reviews of the literature on CTE. ${ }^{28,29}$ Although clinical research on CTE is limited primarily to case reports with neuropathologic confirmation, ${ }^{27}$ the findings to date indicate that this condition may develop years after the head trauma has occurred.

CTE is dissociable from PCS and other acute effects of mTBI. The neurobehavioral effects of CTE, including dysregulation of mood and motor deficits, are also atypical of the chronic effects of a single mTBI. CTE has been reported after repeated subconcussive blows to the head even without history of a concussion-i.e., mTBI associated with altered consciousness or post-traumatic amnesia. Although epidemiologic data on CTE are sparse, host factors, including the presence of the APOE 4 allele, appear to increase vulnerability to development of this condition. ${ }^{28}$
Case reports of professional and amateur athletes ${ }^{30,31}$ in whom CTE developed after repeated concussion have provided impetus to study repeated mTBI from other etiologies, including blast in service members and veterans. Investigation of repetitive mTBI in civilians other than sports concussion is challenged by its relatively low rate of occurrence.

\section{Military mTBl}

mTBI in military personnel differs from civilian mTBI in regard to the population, cause of injury, and risk of repetitive injury. The military population is more homogenous in age, sex, and general health than the civilian population. Blast-related TBI resulting from improvised explosive devices has been reported to be the most frequent mechanism of mTBI during the recent wars in Iraq and Afghanistan. ${ }^{7}$ MacDonald and colleagues, ${ }^{14}$ however, recently found that combined blast and non-blast mechanisms were typically present in wounded warriors who were evacuated to a higher level of hospitalized care after mTBI associated with polytrauma.

In contrast to civilian mTBI, differentiation of PCS associated with mTBI from post-traumatic stress disorder (PTSD) symptoms often complicates assessment of service members and veterans, especially at long intervals after combat-related injury. Postdeployment, self-report data disclosed co-morbid PTSD symptoms at or above threshold severity for clinical diagnosis in $44 \%$ of service members whose mTBI produced loss of consciousness (LOC). ${ }^{27}$ Combat intensity is directly related to both the risk of sustaining mTBI and to development of PTSD. Compared with cross-sectional investigations that enroll service members and veterans months or even years after injury, prospective studies beginning during the subacute phase of $\mathrm{mTBI}^{14}$ are better suited to differentiate the process of recovery after mTBI from the emergence of PTSD.

Recording clinical features of acute mTBI in theater is more difficult than in civilian situations and pressures to return to duty are balanced against indications for medical evacuation to a higher level of care than a field hospital or concussion clinic. Acute intoxication at the time of injury, however, is not a factor in combatrelated $\mathrm{mTBI}$.

Despite distinctive features of blast and non-blast TBI, cognitive sequelae and residual symptoms of civilian and blast mTBI share common features. ${ }^{10}$ Comparison of impaired consciousness, subacute symptoms, and cognitive performance within 72 hours after blast $(n=39)$ or non-blast $(n=38)$ TBI in wounded warriors and military contractors was reported by Luethcke and coworkers. ${ }^{13}$ LOC was more frequently reported after non-blast injury, and a higher proportion of this group had problems with balance, nausea, and vomiting early after injury than the blast group. In contrast, complaints of hearing difficulty were significantly more frequent in the blast-injured group.

Although cognitive processing speed was sensitive to duration of LOC and generally declined from pre-injury baseline performance, it did not differ between blast and non-blast injury groups. Consistent with these subacute data, Belanger and colleagues ${ }^{10}$ found that there were no between-group differences in cognitive performance at about 2 years post-injury between veterans who had sustained blast $(n=61)$-related TBI and those with non-blast ( $n=41)$ TBI on measures of learning, memory, flexibility in cognitive response, and cognitive processing speed. On subdividing each mechanism of injury group into mild versus moderate/severe injury subgroups, however, these investigators did find an interaction of mechanism with severity of injury on a test of visual 
memory. Veterans who had mild blast TBI obtained the best visual memory performance, whereas the moderate/severe blast TBI group had the worst scores. Comparison of the mechanism of injury groups on a self-reported measure of PTSD symptoms disclosed a marginally significant effect, with more severe symptoms in the blast than non-blast groups. The authors postulated that hyperarousal at the time of blast injury may predispose to more persistent PTSD symptoms than is the case with non-blast injury.

\section{Neural mechanisms of mTBI}

Constraints on elucidating the neural mechanisms of mTBI have included animal models that produce focal contusions, rather than multifocal or diffuse axonal injury, which are considered to be the primary mechanisms of mTBI in humans. ${ }^{32-34}$ A subset of clinical studies focused on investigating the effects of diffuse axonal injury ${ }^{35}$ have even excluded patients with acute contusions identified by $\mathrm{CT}$. Although few neuropathological studies of mTBI in humans have been reported, Blumbergs and associates ${ }^{36}$ found evidence of axonal injury in five adults who had sustained mTBI associated with more severe injuries. Consistent with Blumbergs and coworkers, ${ }^{36}$ mTBI denotes a predominantly white matter injury that is on a continuum of severity with moderate to severe brain injuries that impart rotational acceleration to the head, including motor vehicle crashes falls, and sports concussion.

Elucidating the neural mechanisms of mTBI has also been delayed by a lack of prospective, longitudinal brain imaging data in patients beginning in the subacute phase of injury. ${ }^{15}$ In the following paragraphs, it will be apparent that advanced brain imaging and neurophysiological techniques have disclosed residual alteration of brain function at varying intervals after mTBI, even when PCS have resolved and cognitive performance has recovered. As described below, changes in brain activation have been documented during a resting state without imposing any task demands on concussed persons.

A recent DTI study ${ }^{33}$ reported subacute and follow-up imaging data that showed persistent alteration of the microstructure of white matter tracts in the absence of cortical contusions on CT. There is disagreement in the literature, however, about the specific changes on DTI after mTBI and inconsistent evidence that these findings are related to clinical indices of mTBI, cognitive sequelae, or persisting PCS. ${ }^{17,33}$ With evolving imaging protocols, technical advances in scanners, and new approaches to analysis of DTI, ${ }^{37}$ this technique has potential as a biomarker for acute, if not chronic mTBI.

Resting state functional magnetic resonance imaging $(\mathrm{rs}[\mathrm{fMRI}]),{ }^{38,39}$ which is recorded without presenting a task to a subject, has disclosed alteration of functional connectivity of brain regions comprising task related and default mode networks after mTBI. Alteration of neural networks during a resting state has been reported in athletes who were no longer symptomatic asfter a concussion $^{39}$ and in patients studied within the first 2 to 3 weeks after mTBI resulting from various external mechanisms. ${ }^{33}$ Advantages of rsfMRI include mitigation of group differences in performance that may affect brain activation or complicate interpretation of taskrelated fMRI. ${ }^{40}$

Task-related fMRI has also been useful in elucidating the neural effects of mTBI. A seminal study by McAllister and colleagues ${ }^{40}$ showed that the pattern of activation associated with a working memory task in patients within 30 days after mTBI had a different pattern compared with uninjured subjects. mTBI patients exhibited a disproportionate increase in the extent of activation of pre-frontal and parietal regions when the memory load was increased to an intermediate level, whereas a further increase in memory load had a greater effect on activation in the uninjured subjects than the mTBI patients. Despite persistence of PCS at the time of imaging, the mTBI patients' efficiency of working memory was comparable to that of the uninjured subjects. McAllister and coworkers ${ }^{40}$ suggested that this alteration in neuromodulation of brain region recruitment may have resulted from mTBI-induced changes in neurotransmitter levels.

Electroencephalography (EEG) and quantitative EEG (qEEG) have been used in studies of subacute and chronic mTBI. The EEG and qEEG literature is reviewed in this issue by Haneef and associates (see Table of Contents). Subacute focal and diffuse slowing on EEG has been widely reported and related to the duration of post-traumatic amnesia during the first days and initial weeks after mTBI. The reader is referred to the review by Haneef and associates for a synthesis of various EEG characteristics during the acute, subacute, and chronic phases of recovery from mTBI.

Magnetic source imaging (MSI) or magnetoencephalography has also been used to characterize changes in connectivity of various brain regions after mTBI. Zouridakis and colleagues ${ }^{41}$ recorded resting state MSI data from 10 patients with mTBI at 3 months post-injury. Despite normal CT and neurological findings at the time of evaluation in the emergency center, the mTBI patients exhibited reduced long-range connectivity between homologous cortical regions (e.g., left prefrontal with right prefrontal regions) and in long connections such as left anterior with right posterior regions. In contrast, shorter connections were relatively intact compared with MSI data in healthy, uninjured subjects. These preliminary findings raise the possibility that functional connectivity on MSI could represent a "biomarker" for mTBI.

Brain metabolic changes induced by mTBI have been postulated as a neural mechanism for cognitive impairment and PCS, especially during the early phases of recovery from mTBI. Magnetic resonance spectroscopy (MRS) is a noninvasive imaging technique that has demonstrated changes in brain metabolites in collegiate athletes during the subacute phase of recovery from concussion. ${ }^{38}$ Ratios of N-acetyl-aspartate to choline (NAA/Cho) and to creatine (NAA/Cre) were identified in the genu but not the splenium of the corpus callosum of recently concussed athletes. Interestingly, the sizes of these reductions were greater in athletes who had sustained their first concussion compared with players who had a history of previous mTBI.

Biomarkers measured from blood have only recently been identified, holding the promise of diagnosing mTBI when other findings are equivocal because of confounders such as intoxication, multiple traumas, or pre-existing conditions. The development of protein biomarkers, however, has not yet reached a level that permits accurate differentiation of acute mTBI from general trauma.

In this overview, we present a conceptual model of mTBI, discuss methodological issues in mTBI research, efforts to improve the validity of animal models, and critical questions that remain to be addressed.

\section{mTBI in translation}

This Special Issue focuses on two types of translation. The first is between animal models designed to approximate mTBI and the translation to clinical, prospective investigation of patients with mTBI using multimodality brain imaging. As discussed below, reproduction of mTBI in animals similar to the clinical presentation is inherently difficult and arguably unachievable, at least in rodents. At the same time, the experimental studies presented in this Special 
Issue provide evidence of producing some of the acute effects of human mTBI. The second translation emphasized here is between civilian mTBI and mTBI in theater, which also introduces methodological issues about differences between the injury mechanisms of blast and non-blast, despite apparent similarity in the severity and duration of impaired consciousness. In the following section, we present a conceptual model to integrate the mechanisms of clinical mTBI with animal models and to identify similarities and differences between civilian and military MTBI.

The diagnosis of post-concussion syndrome requires persistence of at least three of eight common PCS for at least 3 months, ${ }^{15}$ whereas the more restrictive diagnosis of post-concussion disorder (PCD) also requires that onset of the symptoms occurs after the injury or at least the symptoms worsen relative to pre-injury and disrupt the individual's social role performance. ${ }^{42}$ Acute traumatic stress symptoms are measured within the first month after injury, a period when the diagnosis of acute stress disorder can be made. Although the diagnosis of PTSD can be made only after 1 month, the symptoms can be evaluated using a rating scale at any time after injury. In contrast to pre-injury risk factors, resilience to stress enhances the prospects for resolution of symptoms and reduces the likelihood of secondary conditions such as depression and PTSD. In contrast, exposure to pre-injury stressors, involving the family environment or other traumatic events, also increases vulnerability to development of persistent PCS and possibly PTSD after mTBI and associated trauma.

Trauma history is especially relevant in combat-related mTBI, which is often associated with repeated exposure to life-threatening or otherwise severe stress over a prolonged period. Scales measuring combat exposure are related to the development of PTSD in service members and veterans. ${ }^{43}$ Pre-injury cognitive ability and level of education are indices of cognitive reserve that may buffer the effects of mTBI. Similarly, genetic factors may also have a role in recovery from mTBI.

Taken together with resilience to stress, these individual differences have been postulated to explain interindividual variation in recovery from similar injuries.

\section{mTBI experimental models}

For understanding the pathophysiology of mTBI and for developing neuroprotective treatments, an experimental model would be invaluable. Modeling mTBI in rodents, however, has been problematic. In most cases, the "mild" versions of the common experimental models of TBI have simply been less severe degrees of traumatic injury, and the animals do not necessarily exhibit the clinical characteristics of human mTBI. Several commonly used experimental TBI models include fluid percussion injury (FPI), controlled cortical impact injury (CCI), weight drop, and impact acceleration models. ${ }^{44-47}$ Blast injury induced with a shock tube has also grown in interest to better model military injuries. Mild versions of each of these TBI models have been characterized.

FPI is the oldest, most well-characterized experimental TBI model. The severity of the injury is varied by altering the angle from which the pendulum is dropped. The position of the injury cap can also be varied from a midline (or central) position between bregma and lambda to lateral position over the parietal region lateral to the sagittal suture. The midline FPI is primarily a diffuse injury, while the lateral FPI also has some element of a focal injury, although in the mild versions of the models, these distinctions are less clear. Mild lateral FPI models have been used as a model of concussion. ${ }^{48}$ An FPI generating a pressure of 0.9 atm has been characterized as a mild FPI in mice, causing a recovery of right reflex time of 2-4 minutes, and mild deficits on Morris water maze testing and rotorod testing. ${ }^{49}$

Although axonal injury assessed with amyloid precursor protein staining was limited to the cingulum at $4 \mathrm{~h}$ post-injury, there was evolution of the injury with axonal damage present in the external capsule at 4 and 6 weeks post-injury and in dorsal thalamic nuclei at 6 weeks post-injury. ${ }^{50}$ In rats, an FPI of 1.2 atm has been described as mTBI, producing a recovery of righting reflex at an average $93 \mathrm{sec}$ post-injury, and transient behavioral abnormalities, including a decrease in anxiety assessed by the elevated-plus maze and slower learning on a Morris water maze task when the platform was moved $24 \mathrm{~h}$ after the rats had been trained to find the platform in the opposite quadrant. $^{48}$

With CCI, the severity of injury can be adjusted by varying the impact velocity and time of impact and by varying the amount of brain deformation. A range of injuries from very mild to very severe can be produced. ${ }^{51-53}$ The impact parameters used to produce a mild injury vary widely from study to study, but injuries with minimal neuronal loss have been characterized. In a mild mouse CCI model using $0.2 \mathrm{~mm}$ deformation and velocity of $2.8 \mathrm{~m} / \mathrm{sec}$, little tissue disruption was observed, and fluoro-Jade B stained cells were few and limited to the cortex at the site of impact. ${ }^{54}$ Extensive dendritic degeneration and reduction in the number of synapses were also described in surviving neurons. A closed skull version of the CCI model in mice has also been developed, using impact parameters of $5 \mathrm{~m} / \mathrm{sec}$ and $1 \mathrm{~mm}$ deformation. ${ }^{55}$ With a single closed skull impact, the mice had transient mild deficits on a neuroscore test and small areas of axonal injury.

In a rat CCI model using $0.5 \mathrm{~mm}$ deformation at velocity of $6 \mathrm{~m} /$ sec, mild deficits at 3 days post injury were found on rotarod and Morris water maze testing. ${ }^{52}$ Using $3 \mathrm{~m} / \mathrm{sec}$ velocity and $1 \mathrm{~mm}$ deformation as a mild CCI model in rats, Abel Baki and cowork$\mathrm{ers}^{53}$ found that performance on a conflict active avoidance task was impaired and performance on an active place avoidance task was transiently impaired (day 1 post-injury). In contrast, more severely injured animals $(4 \mathrm{~m} / \mathrm{sec}$ velocity and $2.5 \mathrm{~mm}$ deformation) had persistently impaired performance on the active place avoidance task.

In mice, a closed head injury weight drop model (20-30 g weight dropped $80 \mathrm{~cm}$ onto the anterolateral skull) has been described. ${ }^{56}$ Anxiety assessed with the elevated plus maze was not abnormal. Spatial learning assessed with the Morris water maze task was impaired for up to 90 days post-injury. Non-spatial learning assessed with a passive avoidance task and working memory tested with the swim T-maze task were most impaired at 30 days postinjury.

Mild blast injury in the mouse has been characterized using a shock tube with a membrane rupture pressure of 26.5 pounds per square inch gauge (psig), average static pressure of 9.9 psig, and total pressure of 14.9 psig. ${ }^{57}$ Multi-focal axonal injury occurred, especially in cerebellum and brainstem. Transient deficits in spatial recognition memory using a Y-maze and motor performance on the rotarod test were observed after the mild blast injury. No changes in anxiety level assessed from open field activity occurred after injury.

Overall, the FPI and CCI models have the best characterization over varied injury levels, but the closed head and blast injury models may have more analogy in terms of mechanism of injury to human mTBI. These models also lend themselves more easily to repetitive injury paradigms, which may be also particularly important with mTBI. ${ }^{55,58,59}$ The current mTBI models have the greatest usefulness in studying the underlying pathophysiology of 
brain injury and in studying the vulnerability of the injured brain to second insults. The behavioral characteristics of the models, however, have limited correlation with the major clinical features of mTBI.

The "fencing response" in acute mild to moderate TBI is a translational feature that has been reported in rodent FPI and observed in videos of impacts producing sports concussion in athletes. ${ }^{60}$ This extension of one limb concurrent with flexion of the contralateral limb is associated with longer suppression of the righting reflex in FPI in rats and occurs in two-thirds of sports concussions resulting in LOC. Translational features such as the fencing response may facilitate evaluation of interventions for acute mTBI.

\section{Limitations of current mild injury models}

Experimental mTBI models reproduce some of the sequelae of mTBI that occur in humans, particularly those related to learning and memory. Some impediments remain, however, including that many PCS described in humans are difficult or impossible to assess in rodents, experimental TBI does not involve traumatic stress that often co-exists in human mTBI, and PCS are transient in most patients with mTBI—only a fraction have persistent problems. The latter is a particularly difficult problem to model because the goal in the laboratory is to have a very consistent model in which all animals undergoing a particular injury level have a similar outcome. Pre-injury co-morbidities associated with traumatic injury in patients are also difficult to model.

The PCS that have been commonly assessed after mTBI in rodents are anxiety, depression, balance/coordination problems, and cognitive difficulties. ${ }^{61}$ Anxiety has been assessed using the elevated-plus maze in which a decreased time spent in the open arms of the maze indicates heightened anxiety. Depression has been assessed using a forced swim task in which increased time spent immobile suggests a depression-like behavior. Sensory sensitivity has been assessed with a whisker nuisance task, in which whisker stimulation elicts aggravated behavioral responses after mild FPI. ${ }^{62,63}$ Balance/coordination has been studied with beam balance and walking tasks, and the rotarod task.

Spatial learning and memory has been tested with Morris water maze and other maze tasks. Non-spatial learning has been tested with passive avoidance tests or novel object recognition test. Conflict active avoidance tasks may be particularly sensitive for mTBI. ${ }^{53}$ Working memory can also be tested with Morris water

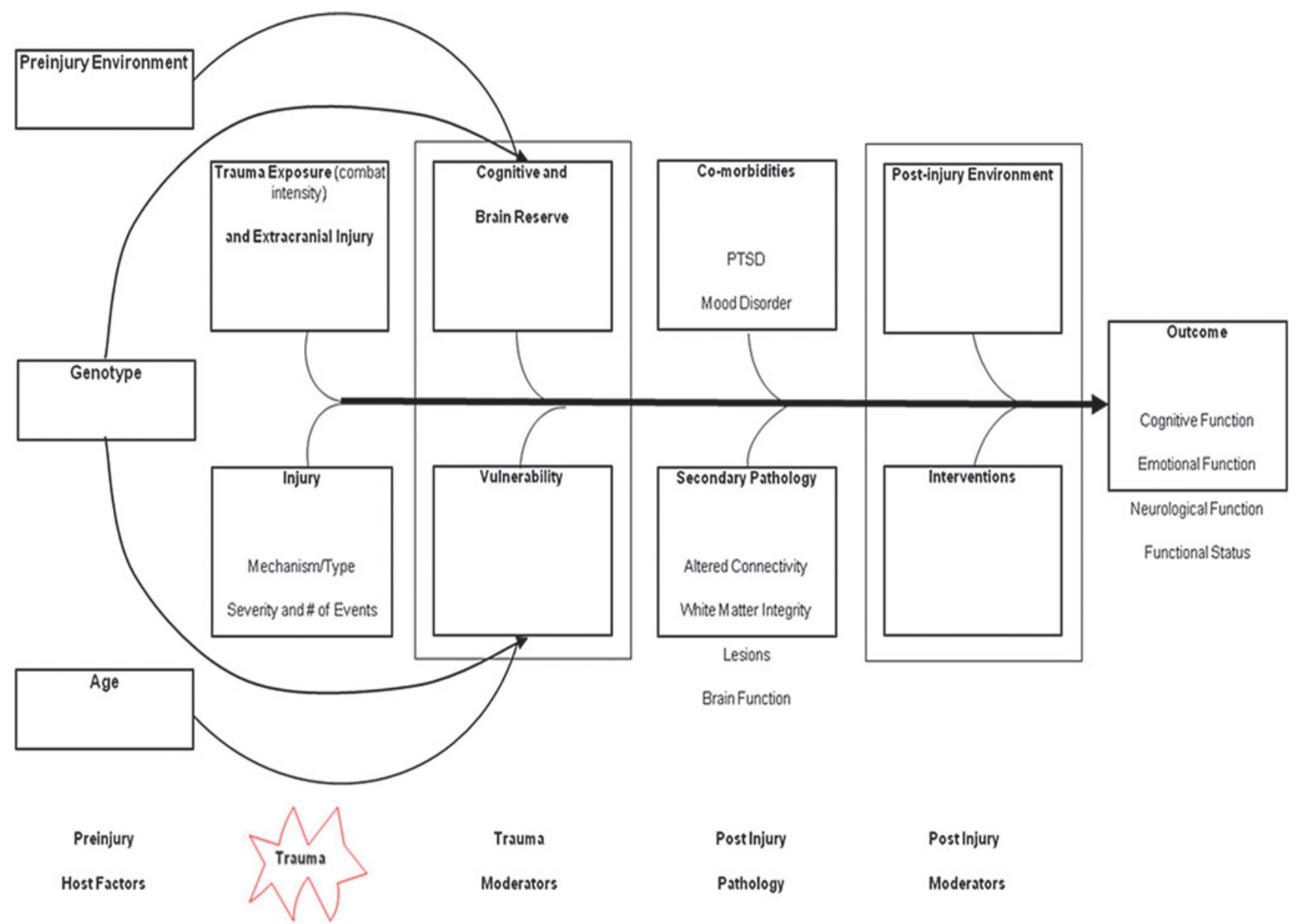

FIG. 1. Conceptual model of mTBI in humans, including pre-injury host factors, moderating effects of environment, injury mechanism and number of injuries, associated trauma exposure, and concomitant injuries. Co-morbid conditions exacerbate the effects of mTBI, whereas a supportive post-injury environment and intervention may mitigate the effects of mTBI. Host factors, including age, genotype, cognitive and brain reserve, may also moderate the effects of mTBI. Adapted from a figure provided by Randall Scheibel, $\mathrm{PhD}$, and Elisabeth Wilde, $\mathrm{PhD}$. 
maze using a modified testing paradigm. Symptoms that are common in humans with mTBI but which cannot be readily assessed in rodents are headache, dizziness, nausea, irritability, fatigue, ringing in ears, visual disturbances, and sleep disorders. Development of more sensitive behavioral tasks for use in mTBI models would help advance the field.

In humans, mTBI symptoms overlap and are inherently linked to PTSD symptoms, especially in combat-related injury. PTSD can be modeled in experimental animals, using fear conditioning. Experimental models of mTBI, however, involve injury under anesthesia and do not generally capture the stress element of a traumatic injury in humans. Other aspects of the experimental paradigm, including transportation and anesthesia, have been shown to alter anxiety level, and these conditions plus sounds of an explosive blast were found to cause an increased serum corticosterone levels and altered biochemistry in amygdale and ventral hippocampus. ${ }^{64}$ These findings suggest that some behavioral characteristics that might be evaluated as effects of mTBI could be confounded by stress in experimental models as well. This aspect needs further study, especially in mTBI models.

Because the symptoms are mild and transient, drug effects are more difficult to assess in mTBI models. It is not clear what improvement in behavioral tasks in a rodent model would indicate a meaningful improvement in humans. Additional work is needed to determine if a more rapid return to normal performance of a rodent behavioral task with an experimental drug treatment indicates that the drug would reduce the severity of PCS in a patient with mTBI.

\section{Summary and Conceptual Model of mTBI}

Figure 1 shows the effects of mTBI and concomitant injuries over time. As represented in Figure 1, exposure to pre-injury stressors involving the family environment or other traumatic events increase vulnerability to development of persistent PCS and possibly PTSD after mTBI and concomitant trauma. Trauma history is especially relevant in combat-related mTBI, which is often associated with repeated exposure to life-threatening events or otherwise severe stress over a prolonged period. Scales measuring combat exposure are related to the development of PTSD in service members and veterans. ${ }^{43}$ Pre-injury cognitive ability and level of education are indices of cognitive reserve that may buffer the effects of mTBI. As seen in Figure 1, genetic factors may also have a role in recovery from mTBI.

Taken together with resilience to stress, these individual differences have been postulated to explain interindividual variation in recovery from similar injuries.

Past mTBI could have additive effects with the current mTBI, ${ }^{24,65}$ and co-morbidities, both medical and psychiatric, can affect recovery. As seen in Figure 1, the post-injury environment could moderate recovery. A supportive environment in which the person gradually resumes activities may enhance resolution of PCS, whereas premature demands to resume full responsibilities despite residual PCS and cognitive deficit may lead to secondary anxiety and depression ${ }^{66}$ and prolong secondary brain insult, such as inflammation and excitotoxicity. Empirical testing of current regimens, such as "cognitive rest" with gradual resumption of physical training, however, is needed to determine the optimal postinjury environment.

Moderation of these effects by host factors is also represented in civilians and service members. Presence of pre-injury psychiatric conditions, particularly anxiety-related disorders, is predictive of persistent PCS after mTBI, ${ }^{1}$ including cognitive and somatic symptoms in addition to emotional manifestations such as depression, anxiety, and stress-related symptoms. PTSD is a frequent co-morbidity that often intensifies over time in military mTBI, but it is less common in civilian injuries. Depression is a common comorbidity or secondary condition in both civilian and military mTBI, however. As noted earlier, the military population at risk for mTBI is more homogeneous than civilians in regard to demographic features, but the combined presence of blast and non-blast mechanisms complicates the characterization of military mTBI.

\section{Directions for Research}

mTBI sequelae often compromise performance of work and leisure activities for periods of weeks to months, and the economic burden of these injuries is enormous because of the high incidence of this condition. In civilian mTBI, the presence of pre-injury conditions, results of brief assessment during the subacute phase, and resting state fMRI are potentially useful in identifying a highrisk subgroup of patients in whom an intervention or at least monitoring progress could mitigate secondary conditions such as post-concussion syndrome. A similar strategy may also be useful in military mTBI. Development of an effective, early intervention to mitigate the sequelae of mTBI would be facilitated by translational research and clinical trials to evaluate treatments that are emerging from animal model research. Multidisciplinary collaboration and multicenter clinical trials would enhance this proposed program of research. Advances in brain imaging and biomarkers may facilitate detection of mTBI and its differentiation from general trauma and other causes of altered consciousness. Further research is necessary to achieve this goal, however. Finally, research is also needed to refine the predictive value of advanced brain imaging techniques and emerging biomarkers.

\section{Acknowledgments}

This research was supported by Grant 5P01NS056202 from the National Institute of Neurological Disorders and Stroke, by award W81XWH-08-2-0133 PT074693P2 from the Department of Defense (Post-Traumatic Stress Disorder and Traumatic Brain Injury (PTSD/TBI) Research Program), and award 26244 from The Center of Excellence for Traumatic Brain Injury Award from the Department of Veterans Affairs. The content is solely the responsibility of the authors and does not necessarily represent the official views of the National Institute of Neurological Disorders and Stroke, the National Institutes of Health, the Department of Veterans Affairs, or the Department of Defense.

\section{Author Disclosure Statement}

No competing financial interests exist.

\section{References}

1. Ponsford, J., Cameron, P., Fitzgerald, M., Grant, M., Mikocka-Walus, A. (2011). Long-term outcomes after uncomplicated mild traumatic brain injury: a comparison with trauma controls. J. Neurotrauma 28, 937-946.

2. IOM (Institute of Medicine), Gulf War and Health, Volume 7. (2009). Long-term Consequences of Traumatic Brain Injury. The National Academic Press: Washington, DC.

3. Cernak, I., and Noble-Haeusslein, L.J. (2010). Traumatic brain injury: an overview of pathobiology with emphasis on military populations. J. Cereb. Blood Flow Metab. 30, 255-266.

4. Belanger, H.G., and Vanderploeg, R.D. (2005). The neuropsychological impact of sports-related concussion: a meta-analysis. J. Int. Neuropscyhol. Soc. 11, 345-357. 
5. McCrea, M., Barr, W.B., Guskiewicz, K., Randolph, C., Marshall S.W., Cantu, R., Onate, J.A., and Kelly, J.P. (2005). Standard regression-based methods for measuring recovery after sport-related concussion. J. Int. Neuropscyhol. Soc. 11, 58-69.

6. Faul, M., Xu, L., Wald, MM, and Coronado, V.G. (2010). Traumatic Brain Injury in the United States: Emergency Department Visits, Hospitalizations and Deaths 2002-2006. Centers for Disease Control and Prevention, National Center for Injury Prevention and Control: Atlanta, GA.

7. Terrio, H., Brenner, L.A., Ivins, B.J., Cho, J.M., Helmick, K., Schwab, K., Scally, K., Bretthauer, R., and Warden, D. (2009). Traumatic brain injury screening: preliminary findings in a US Army Brigade Combat Team. J. Head Trauma Rehabil. 24, 14-23.

8. Hoge, C.W., Castro, C.A., Messer, S.C., McGurk, D., Cltting, D.I., and Koffman, R.L. (2004). Combat duty in Iraq and Afghanistan, mental health problems, and barriers to care. N. Engl. J. Med. 351, 13-22.

9. Levin, H.S., Wilde, E., Troyanskaya, M., Petersen, N.J., Scheibel, R., Newsome, M. Radaideh, M., Wu, T., Yallampalli, R., Chu, Z., and Li, X. (2010). Diffusion tensor imaging of mild to moderate blast-related traumatic brain injury and its sequelae. J. Neurotrauma 27, 683-694.

10. Belanger, H.G., Kretzmer, T., Yoash-Gantz, R., Pickett, T., and Tupler, L.A. (2009). Cognitive sequelae of blast-related versus other mechanisms of brain trauma. J. Int. Neuropsychol. Soc. 15, 1-8.

11. Davenport, N.D., Lim, K.O., Armstrong, M.T., and Sponheim, S.R. (2012). Diffuse and spatially variable white matter disruptions are associated with blast-related mild traumatic brain injury. Neuroimage 59, 2017-2024.

12. Morey, R.A., Haswell, C.C., Selgrade, E.S., Massoglia, D., Liu, C., Weiner, J., Marx, C.E., Cernak, I., and McCarthy, G; MIRECC Work Group. (2012). Effects of chronic mild traumatic brain injury on white matter integrity in Iraq and Afghanistan war veterans. Hum. Brain Mapp. E-pub ahead of print.

13. Luethcke, C.A., Bryan, C.J., Morrow, C.E., and Isler, W.C. (2011). Comparison of concussive symptoms, cognitive performance, and psychological symptoms between acute blast-versus nonblast-induced mild traumatic brain injury. J. Int. Neuropscyhol. Soc. 17, 36-45.

14. Mac Donald, C.L., Johnson, A.M., Cooper, D., Nelson, E.C., Werner, N.J., Shimony, J.S., Snyder, A.Z., Raichle, M.E., Witherow, J.R., Fang, R., Flaherty, S.F., and Brody, D.L.. (2011). Detection of blastrelated traumatic brain injury in U.S. military personnel. N. Engl. J. Med. 364, 2091-2100

15. Carroll, L.J., Cassidy, J.D., Peloso, P.M., Borg, J., von Holst, H., Holm, L., Paniak, C., and Pépin M. (2004). Prognosis for mild traumatic brain injury: results of the WHO Collaborating Centre Task Force on Mild Traumatic Brain Injury. J. Rehabil. Med. Suppl 43, 84-105.

16. Alexander, M.P. (1995). Mild traumatic brain injury: pathophysiology, natural history, and clinical management. Neurology 45, 1253-1260.

17. Iverson, G.L., Lovell, M.R., and Collins, M.W. (2005). Validity of ImPACT for measuring processing speed following sports-related concussion. J. Clin. Exp. Neuropsychol. 27, 683-689.

18. Walsh, J.M., Flegel, R., Atkins, R., Cangianelli, L.A., Cooper, C., Welsh, C., and Kerns, T.J. (2005). Drug and alcohol use among drivers admitted to a Level-1 trauma center. Accid. Anal Prev. 37, 894-901.

19. Dikmen, S., et al. (1995). Neuropsychological outcome at 1 year post head injury. Neuropsychology 9, 80 .

20. Boake, C., McCauley, S.R., Pedroza, C., Levin, H.S., Brown, S.A., and Brundage, S.I. (2005). Lost productive work time after mild to moderate traumatic brain injury with and without hospitalization. Neurosurgery 56, 994-1003.

21. Dikmen, S.S., Ross, B.L., Machamer, J.E., and Temkin, N.R.. (1995). One year psychosocial outcome in head injury. J. Int. Neuropsychol. Soc. 1, 67-77.

22. Williams, D.H., Levin, H.S., and Eisenberg, H.M. (1990). Mild head injury classification. Neurosurgery 27, 422-428.

23. Crisco, J.J., Wilcox, B.J., Machan, J.T., McAllister, T.W., Duhaime, A.C., Duma, S.M., Rowson, S., Beckwith, J.G., Chu, J.J., and Greenwald, R.M. (2012). Magnitude of head impact exposures in individual collegiate football players. J. Appl. Biomech. 28, 174-183.

24. Gronwall, D., and Wrightson, P. (1975). Cumulative effect of concussion. Lancet 2, 995-997.

25. Hoge, C.W., McGurk, D., Thomas, J.L., Cox, A.L., Engel, C.C., and Castro, C.A. (2008). Mild traumatic brain injury in U.S. soldiers returning from Iraq. N. Engl. J. Med. 358, 453-463.

26. McAllister, T.W., Flashman, L.A., Maerlender, A., Greenwald, R.M., Beckwigth, J.G., Tosteson, T.D., Crisco, J.J., Brolinson, P.G., Duma,
S.M., Duhaime, A.C., Grove, M.R., Turco, J.H. (2012). Cognitive effects of one season of head impacts in a cohort of collegiate contact sport athletes. Neurology 78, 1777-1784.

27. McKee, A.C., Cantu, R.C., Nowinski, C.J., Hedley-Whyte, E.T., Gavett,ds.E., Busdon, A.E., Santini, V.E., Lee, H.S., Kubilus, C.A., Stern, R.A. (2009). Chronic traumatic encephalopathy in athletes: progressive tauopathy after repetitive head injury. J. Neuropathol. Exp. Neurol. 68, 709-735.

28. Baugh, C.M., Stamm, J.M., Riley, D.O., Gavett, B.E., Shenton, M.E. Lin, A., Nowinski, C.J., Cantu, R.C., McKee, A.C., and Stern, R.A. (2012). Chronic traumatic encephalopathy: neurodegeneration following repetitive concussive and subconcussive brain trauma. Brain Imaging Behav. 6, 244-254.

29. Stern, S. (2011). Observing and recording neurological dysfunction. Emerg Nurse 18, 28-31.

30. Omalu, B.I., DeKosky, S.T., Hamilton, R.L., Minster, R.L., Kamboh, M.I., Shakir, A.M., and Wecht, C.H. (2006). Chronic traumatic encephalopathy in a national football league player: part II. Neurosurgery 59, 1086-1093.

31. Omalu, B.I., DeKosky, S.T., Minster, R.L., Kamboh, M.I., Hamilton, R.L., and Wecht, C.H. (2005). Chronic traumatic encephalopathy in a National Football League player. Neurosurgery 57, 128-134.

32. Browne, K.D., Chen, X.H., Meaney, D.F., and Smith, D.H. (2011). Mild traumatic brain injury and diffuse axonal injury in swine. J. Neurotrauma 28, 1747-1755.

33. Mayer, A.R., Yang, Z., Yeo, R.A., Pena, A, Ling, J.M., Mannell, M.V., Stippler, M., and Mojtahed, K. (2012). A functional MRI study of multimodal selective attention following mild traumatic brain injury. Brain Imaging Behav. 6, 343-354.

34. Meythaler, J.M., Peduzzi, J.D., Eleftheriou, E., and Novack, T.A. (2001). Current concepts: diffuse axonal injury-associated traumatic brain injury. Arch. Phys. Med. Rehabil. 82, 1461-1471.

35. Levin, H.S., Mattis, S., Ruff, R.M., Eisenberg, H.M., Marshall, L.F., Tabaddor, K., High, W.M., Jr., and Frankowski, R.F. (1987). Neurobehavioral outcome following minor head injury: a three-center study. J. Neurosurg. 66, 234-243.

36. Blumbergs, P.C., Scott, G., Manavis, J., Wainwright, H., Simpson, D.A., and McLean, A.J. (1995).Topography of axonal injury as defined by amyloid precursor protein and the sector scoring method in mild and severe closed head injury. J. Neurotrauma 12, $565-572$

37. White, T., Schmidt, M., and Karatekin, C. (2009). White matter 'potholes' in early-onset schizophrenia: a new approach to evaluate white matter microstructure using diffusion tensor imaging. Psychiatry Res. 174, 110-115.

38. Johnson, B., Zhang, K., Gay, M., Horovitz, S., Hallett, M., Sebastianelli, W., and Slobounov, S. (2012). Alteration of brain default network in subacute phase of injury in concussed individuals: restingstate fMRI study. Neuroimage 59, 511-518.

39. Slobounov, S.M., Zhang, K., Pennell, D., Ray, W., Johnson, B., and Sebastianelli, W. (2010). Functional abnormalities in normally appearing athletes following mild traumatic brain injury: a functional MRI study. Exp. Brain Res. 202, 341-354.

40. McAllister, T.W., Saykin, A.J., Flashman, L.A., Sparling, M.B., Johnson, S.C., Guerin, S.J., Mamourian, A.C., Weaver, J.B., and Yanofsky, N. (1999). Brain activation during working memory 1 month after mild traumatic brain injury: a functional MRI study. Neurology 53, 1300-1308.

41. Zouridakis, G., Udit Patidar, N.S., Rezaie, R., Castillo, E.M., Levin, H.S., Papanicolaou, A.C. (2012). Functional connectivity changes in mild traumatic brain injury assessed using magnetoencephalography. J. Mech. Med. Biol. 12, 1240006.

42. American Psychiatric Association. (2000). Diagnostic and Statistical Manual of Mental Disorders. American Psychiatric Association: Washington, DC

43. Vasterling, J.J., Verfaellie, M., and Sullivan, K.D. (2009). Mild traumatic brain injury and posttraumatic stress disorder in returning veterans: perspectives from cognitive neuroscience. Clin. Psychol. Rev. 29, 674-684.

44. O'Connor, W.T., Smyth, A., and Gilchrist, M.D. (2011). Animal models of traumatic brain injury: a critical evaluation. Pharmacol. Ther. 130, 106-113.

45. Dixon, C.E., Clifton, G.L., Lighthall, J.W., Yaghmai, A.A., and Hayes, R.L. (1991). A controlled cortical impact model of traumatic brain injury in the rat. J. Neurosci. Methods 39, 253-262. 
46. Foda, M.A., and Marmarou, A. (1994). A new model of diffuse brain injury in rats. Part II: Morphological characterization. J. Neurosurg. 80, 301-313.

47. Marmarou, A., Foda,M.A., van den Brink, W., Campbell, J., Kita, H., and Demetriadou, K. (1994). A new model of diffuse brain injury in rats. Part I: Pathophysiology and biomechanics. J. Neurosurg. 80, 291-300.

48. Shultz, S.R., MacFabe, D.F., Foley, K.A., Taylor, R., and Cain, D.P. (2011). A single mild fluid percussion injury induces short-term behavioral and neuropathological changes in the Long-Evans rat: support for an animal model of concussion. Behav. Brain Res. 224, 326-335.

49. Alder, J., Fujioka, W., Lifshitz, J., Crockett, D.P., and Thakker-Varia, S. (2011). Lateral fluid percussion: model of traumatic brain injury in mice. J. Vis. Exp. 22, (54).

50. Spain, A., Daumas, S., Lifshitz, J., Rhodes, J., Andrews, P. J., Horsburgh, K., and Fowler, J.H.. (2010). Mild fluid percussion injury in mice produces evolving selective axonal pathology and cognitive deficits relevant to human brain injury. J. Neurotrauma 27, 14291438 .

51. Goodman, J.C., Cherian, L., Bryan, R.M., Jr., and Robertson, C.S. (1994). Lateral cortical impact injury in rats: pathologic effects of varying cortical compression and impact velocity. J. Neurotrauma 11 , 587-597.

52. Yu, S., Kaneko, Y., Bae, E., Stahl, C.E., Wang, Y., van Loveren, H., Sanberg, P.R., Borlongan, C.V. (2009). Severity of controlled cortical impact traumatic brain injury in rats and mice dictates degree of behavioral deficits. Brain Res. 1287, 157-163.

53. Abdel Baki, S.G., Kao, H.Y., Kelemen, E., Fenton, A.A., and Bergold, P.J. ( 2009).A hierarchy of neurobehavioral tasks discriminates between mild and moderate brain injury in rats. Brain Res. 1280, 98-106.

54. Gao, X., and Chen, J. (2011). Mild traumatic brain injury results in extensive neuronal degeneration in the cerebral cortex. J. Neuropathol. Exp. Neurol. 70, 183-191.

55. Laurer, H.L., Bareyre, F.M., Lee, V.M., Troojanowski, J.Q., Longhi, L., Hoover, R., Saatman, K.E., Raghupathi, R., Hoshino, S., Grady, M.S., and McIntosh, T.K. (2001). Mild head injury increasing the brain's vulnerability to a second concussive impact. J. Neurosurg. 95, 859-870.

56. Zohar, O., Rubovitch, V., Milman, A., Schreiber, S., and Pick, C.G. (2011). Behavioral consequences of minimal traumatic brain injury in mice. Acta Neurobiol. Exp. (Wars) 71, 36-45.

57. Koliatsos, V.E., Cernak, I., Xu, L., Song, Y., Savonenko, A., Crain, B.J., Eberhart, C.G., Frangakis, C.E., Meinikova, T., Kim, H., and
Lee, D. (2011). A mouse model of blast injury to brain: initial pathological, neuropathological, and behavioral characterization. J. Neuropathol. Exp. Neurol. 70, 399-416.

58. Kane, M.J., Angoa-Pérez, M., Briggs, D.I., Viano, D.C., Kreipke, C.W., and Kuhn, D.M. (2012). A mouse model of human repetitive mild traumatic brain injury. J. Neurosci. Methods 203, 41-49.

59. Wang, Z., Jia, X., Liang, P., Qi, Z., Yang, Y., Zhou, W., and Li, K. (2012). Changes in thalamus connectivity in mild cognitive impairment: evidence from resting state fMRI. Eur. J. Radiol. 81, 277-285.

60. Hosseini, A.H., and Lifshitz, J. (2009). Brain injury forces of moderate magnitude elicit the fencing response. Med. Sci. Sports Exerc. 41, 1687-1697.

61. Fujimoto, S.T.,Longhi, L., Saatman, K.E., Conte, V., Stocchetti, N., and McIntosh, T.K. (2004). Motor and cognitive function evaluation following experimental traumatic brain injury. Neurosci. Biobehav. Rev. 28, 365-378.

62. Learoyd, A.E., and Lifshitz, J. (2012). Comparison of rat sensory behavioral tasks to detect somatosensory morbidity after diffuse braininjury. Behav. Brain Res. 226, 197-204.

63. McNamara, K.C., Lisembee, A.M., and Lifshitz, J. (2010).The whisker nuisance task identifies a late-onset, persistent sensory sensitivity in diffuse brain-injured rats. J. Neurotrauma 27, 695-706.

64. Kamnaksh, A., Kovesdi, E., Kwon, S.K., Wingo, D., Ahmed, F., Grunberg, N.E., Long, J., and Agoston, D.V. Factors affecting blast traumatic brain injury. J. Neurotrauma (2011). 28, 2145-2153.

65. Johnson, V.E., Stewart, W., and Smith, D.H. (2010). Traumatic brain injury and amyloid-beta pathology: a link to Alzheimer's disease? Nat. Rev. Neurosci. 11, 361-370.

66. Levin, H.S., McCauley, S.R., Josic, C.P., Boake, C., B rown, S.A., Goodman, H.S., Merritt, S.G., and Brundage, S.I. (2005) Predicting depression following mild traumatic brain injury. Arch. Gen. Psychiatry. $62,523-528$.

Address correspondence to:

Harvey S. Levin, PhD

Department of Physical Medicine and Rehabilitation

Baylor College of Medicine

1709 Dryden Road, Suite 1200

Houston, TX 77030

E-mail: hlevin@bcm.edu 